\title{
Ceftriaxone-induced Neurotoxicity in a Patient after Pancreas-Kidney Transplantation
}

\author{
Hideharu Hagiya ${ }^{1}$, Koji Miyawaki ${ }^{2}$, Norihisa Yamamoto ${ }^{1}$, Hisao Yoshida ${ }^{1}$, \\ Akihiro Kitagawa ${ }^{3}$, Tadafumi Asaoka ${ }^{3}$, Hidetoshi Eguchi ${ }^{3}$, \\ Yukihiro Akeda ${ }^{1}$ and Kazunori Tomono ${ }^{1}$
}

\begin{abstract}
:
Ceftriaxone is a widely used third-generation cephalosporin showing advantageous pharmacokinetic properties and a broad antimicrobial spectrum. We herein report a case of ceftriaxone-induced neurotoxicity in a 56year-old man on hemodialysis. Seven days after initiating high-dose ceftriaxone, the patient developed impaired consciousness along with facial myoclonus and sporadic phonation. The symptoms clearly disappeared shortly after withdrawal of the drug. Ceftriaxone is considered a safe antibiotic for patients with renal insufficiency, since it is excreted via both haptic and renal pathways. Physicians should note that antibioticassociated encephalopathy may develop in patients administered ceftriaxone, especially in those complicated with renal dysfunction.
\end{abstract}

Key words: antibiotic-associated encephalopathy, ceftriaxone, chronic kidney disease, hemodialysis, nonconvulsive status epilepticus, transplantation

(Intern Med 56: 3103-3107, 2017)

(DOI: 10.2169/internalmedicine.8774-16)

\section{Introduction}

Ceftriaxone (CTRX) is a common antibiotic frequently used in both in- and outpatients. The pharmacological advantages of CTRX include a broad microbial spectrum, long half-life, and good penetration into systemic tissues, including the central nervous system. In contrast to other betalactams, CTRX is metabolized at the liver, and a renal dose adjustment is usually not necessary. In addition, side effects and drug interactions are uncommon. Therefore, it is generally assumed that CTRX can be safely administered to elderly patients, including those with chronic kidney disease (1).

Following antimicrobial treatments, some patients may develop antibiotic-associated encephalopathy (AAE). Among beta-lactams, cefepime, a fourth-generation cephalosporin, is known to cause encephalopathy, particularly in elderly patients with renal insufficiency $(2,3)$. According to a previ- ous review, CTRX is listed as a low-to-medium-risk agent based on its structural characteristics $(4,5)$; however, CTRX-associated encephalopathy has rarely been reported. To our knowledge, only 10 such cases have been documented in the literature to date (6-11). In this report, we describe another case of CTRX-induced neurotoxicity complicated by involuntary facial movements.

\section{Case Report}

The patient was a 56-year-old man (dry body weight: $50.5 \mathrm{~kg}$ ). He had undergone simultaneous pancreas-kidney transplantation five years earlier, but he was on hemodialysis due to graft failure. One day, he was found to have a low blood pressure and decreased oxygen saturation at a hemodialysis clinic and was immediately transferred to our hospital as an emergency case. On admission, he was alert but in a state of shock. He reported pain in his left chest, which was intensified by deep breaths and dry cough. The patient

${ }^{1}$ Department of Infection Control and Prevention, Osaka University Graduate School of Medicine, Japan, ${ }^{2}$ Department of Pharmacy, Osaka University Hospital, Japan and ${ }^{3}$ Department of Gastroenterological Surgery, Osaka University Graduate School of Medicine, Japan Received: December 27, 2016; Accepted: March 27, 2017; Advance Publication by J-STAGE: September 25, 2017 Correspondence to Dr. Hideharu Hagiya, highgear@hp-infect.med.osaka-u.ac.jp 


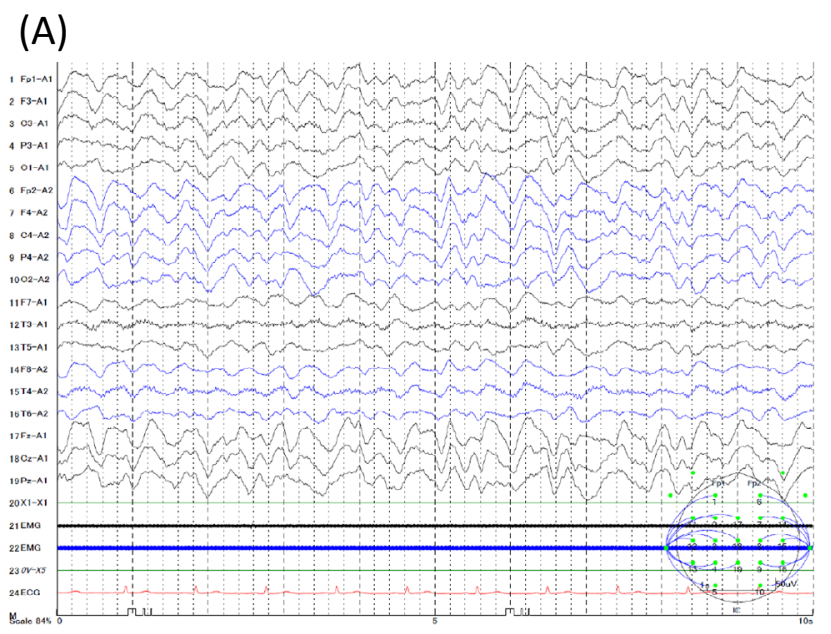

(B)

Figure 1. Electroencephalogram (EEG). (A) EEG on day 18. The patient manifested impaired consciousness. Generalized moderate-to-high-amplitude epileptiform bursts continued throughout the test. (B) EEG on day 28. Three days after the patient recovered from unconsciousness. The abnormal spikes completely disappeared, and alpha waves emerged mainly at the occipital lobes.

was prescribed prednisolone (2.5 $\mathrm{mg}$ per day), cyclosporine (120 mg per day), mycophenolate mofetil (500 mg per day), and everolimus (1 mg per day). He had no history of neurological disorders or brain surgery. Laboratory results showed elevated serum C-reactive protein levels (CRP, $21.14 \mathrm{mg}$ / dL). A chest radiograph demonstrated permeability decay in his left lower lung, and computed tomography (CT) of his chest revealed diffuse consolidation in the left lower lobe. The patient was diagnosed with septic shock secondary to pneumonia and pleurisy and was hospitalized at an emergency center. Treatment was started empirically with renal doses of meropenem and levofloxacin.

Three days after hospitalization, the patient was moved to a general ward. Although a bacterial culture of his sputum was unremarkable, antibiotic therapy was switched to CTRX (2 g q12h) and minocycline (100 mg q12h) on day 6. Despite antibiotic treatment, the pleural effusion increased, and he underwent paracentesis of the pleural cavity on day 8 . The pleural fluid was clear, and a bacterial culture was negative. Nevertheless, the levels of serum inflammatory markers and his body temperature remained high.

On admission day 13, the patient started to become disoriented and agitated. At that time, he was afebrile, and laboratory data showed the serum CRP level to be $11.1 \mathrm{mg}$ / $\mathrm{dL}$; otherwise, there were no markedly abnormal values. On the same day, continuous air leakage emerged. Chest CT revealed a newly formed pulmonary cavity in the left lower lobe, indicating pulmonary suppuration and a subsequent thoracic empyema. No explanation was found for the patient's altered consciousness, but the patient was subjected to emergency thoracoscopic surgery. Although there was no apparent purulent effusion, pus was found inside the cavity. Postoperatively, the patient was extubated and admitted to an intensive-care unit. The administration of CTRX was continued in combination with levofloxacin (250 mg q48h) and clindamycin (600 mg q6h). Before the operation, he had undergone intermittent hemodialysis three times per week, which was changed to continuous hemodiafiltration postoperatively.

Even after the discontinuation of sedative drugs, his consciousness remained impaired. The patient's conscious state was mostly at E2V2M5 on the Glasgow Coma Scale, but occasionally, he was unresponsive to stimuli or seemed to be confused or agitated. In addition, involuntary facial movements, including rhythmic contractions of the orbicularis oculi muscles and tongue were observed (Supplementary material). Sporadic phonation seemed to be triggered by dispersive contractions of laryngeal muscles. We did not observe convulsion or athetosis. CT and magnetic resonance imaging (MRI) of his head did not reveal any abnormalities; however, an electroencephalogram (EEG) showed bursts of generalized, high-voltage slow-wave activity throughout the measurement period (Fig. 1A). The differential diagnosis included encephalopathy caused by metabolic, renal, and drug-related etiologies, delirium, and a psychogenic reaction. The levels of serum electrolytes, liver function, thyroid function, thiamine, and ammonia were all within normal ranges, while the serum levels of total protein and albumin declined to 5.0 and $2.4 \mathrm{~g} / \mathrm{dL}$, respectively. A cerebrospinal fluid examination showed normal findings: initial pressure, $16 \mathrm{cmH}_{2}$ O, cells $1 / \mu \mathrm{L}$, protein $36 \mathrm{mg} / \mathrm{dL}$, and glucose $67 \mathrm{mg} / \mathrm{dL}$. Therefore, an adverse drug effect remained a potential cause of the impaired consciousness. Throughout the clinical course, the serum level of cyclosporine was closely monitored and maintained in an appropriate range.

On reviewing the patient's course, a high dose of CTRX was continuously administered for 7 days before the patient fell unconscious (usual dose: 1 to $2 \mathrm{~g}$ per $24 \mathrm{~h}$ ). At this point, CTRX-induced neurotoxicity was suspected. The dosage of CTRX was reduced to $2 \mathrm{~g}$ per day on day 18 , but the 


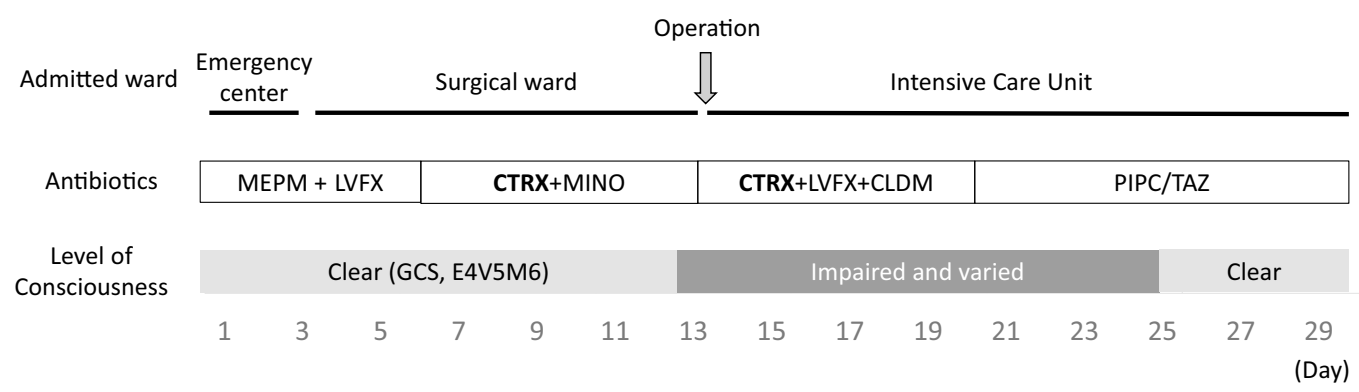

Figure 2. Clinical course of the presented patient. Seven days after initiating CTRX, the patient developed impaired consciousness (day 13). He remained unconscious postoperatively but regained consciousness to a full level (GCS, E4V5M6) 5 days after the cessation of CTRX treatment. Aside from antibiotics, the patient did not receive any drugs continuously after hospitalization. CLDM: clindamycin, CTRX: ceftriaxone, GCS: Glasgow Coma Scale, LVFX: levofloxacin, MEPM: meropenem, MINO: minocycline, PIPC/TAZ: piperacillin/tazobactam

patient's condition did not improve. Subsequently, CTRX was discontinued and switched for piperacillin/tazobactam (2.25 g q8h) on day 20. Five days after the cessation of treatment with CTRX, the patient recovered completely. He was still slightly febrile $\left(37.6^{\circ} \mathrm{C}\right)$, but his general condition and vital signs were stable, and his serum CRP level decreased to $3.5 \mathrm{mg} / \mathrm{dL}$. A follow-up EEG showed no abnormal patterns (Fig. 1B), and no neurological symptoms recurred until his discharge from the hospital. The patient's clinical course is illustrated in Fig. 2.

\section{Discussion}

We encountered a case of CTRX-associated neurotoxicity occurring in a patient after pancreas-kidney transplantation. The onset and resolution of the neurological symptoms appeared to be closely related to the initiation and discontinuation of CTRX administration, and the EEG findings were similar to previous cases $(6,9)$. Although one or more of the other drugs concurrently administered with CTRX (cyclosporine, meropenem, levofloxacin, and minocycline) might have potentially caused the encephalopathy, the influence of any of these drugs seemed unlikely, given the clinical course.

Neurological complications are relatively common after solid organ transplantations, appearing in approximately one-third of such patients (12). Frequent etiologies include immunosuppressive agents (e.g. calcineurin inhibitors), central nervous system infections, and encephalopathy. In particular, the differential diagnosis of neural involvement in the late phase ( $>6$ months) includes viral (Herpes simplex virus, Cytomegalovirus, Epstein-Barr virus, Varicella-zoster virus, and progressive multifocal leukoencephalopathy by JC polyomavirus), fungal (Cryptococcus neoformans, Aspergillus spp., and Mucor), and parasitic (Toxoplasma gondii, Amebiasis, Strongyloides stercoralis) infections (13). In addition, neoplastic etiologies such as post-transplantation lymphoproliferative disorder should also be considered.

Table summarizes the present and previous cases of
CTRX-induced neurotoxicity (6-11). Notably, elderly patients with chronic kidney disease are at particularly high risk for drug-associated encephalopathy. Of the 11 cases, 8 (72.7\%) were over 60 years of age. In addition, four had chronic kidney disease, and half of the patients were on either hemodialysis or peritoneal dialysis. Renal insufficiency is in general a common factor for AAE; $25 \%$ of all cases of AAE and over $70 \%$ of cephalosporin-associated cases have been associated with renal insufficiency (14). A possible reason for the association between renal insufficiency and AAE is impaired biliary excretion in patients with renal dysfunction (5). Impaired biliary excretion may be responsible for the delayed elimination of CTRX, and a subsequently higher serum concentration of the drug can trigger the neurotoxicity.

An excessive dosage can be another predisposing factor for cephalosporin-associated neurotoxicity (15-17). However, the dosages of CTRX administered in the reported cases were mostly normal $(\leq 2 \mathrm{~g}$ per day in 8 of 10 adult cases in Table). Thus, the relationship between overdose of the drug and neurotoxicity is uncertain. As few or no reports on the previous CTRX-associated encephalopathy measured the serum concentration of the drug, the association between the serum concentration and neurotoxicity is inconclusive.

Although the pathophysiological mechanisms of cephalosporin-associated neurotoxicity have yet to be fully elucidated, beta-lactam associated encephalopathy is believed to be related to the competitive inhibition of $\gamma$ aminobutyric acid in brain tissues (15). CTRX penetrates efficiently into the central nervous system and can trigger increased neural excitability even at normal dosages.

Once recognized, AAE is easily treatable by discontinuing the offending drugs. However, the early diagnosis of CTRXinduced neurotoxicity is challenging for three reasons. First, many clinicians are unaware of this rare adverse effect related to CTRX. Second, the clinical presentations may vary among patients; reported symptoms include altered mental status (6 cases), choreoathetosis (4 cases), and myoclonus (4 cases) (Table). Interestingly, our patient manifested facial 
Table. Summary of Reported Cases of Ceftriaxone (CTRX)-induced Neurotoxicity.

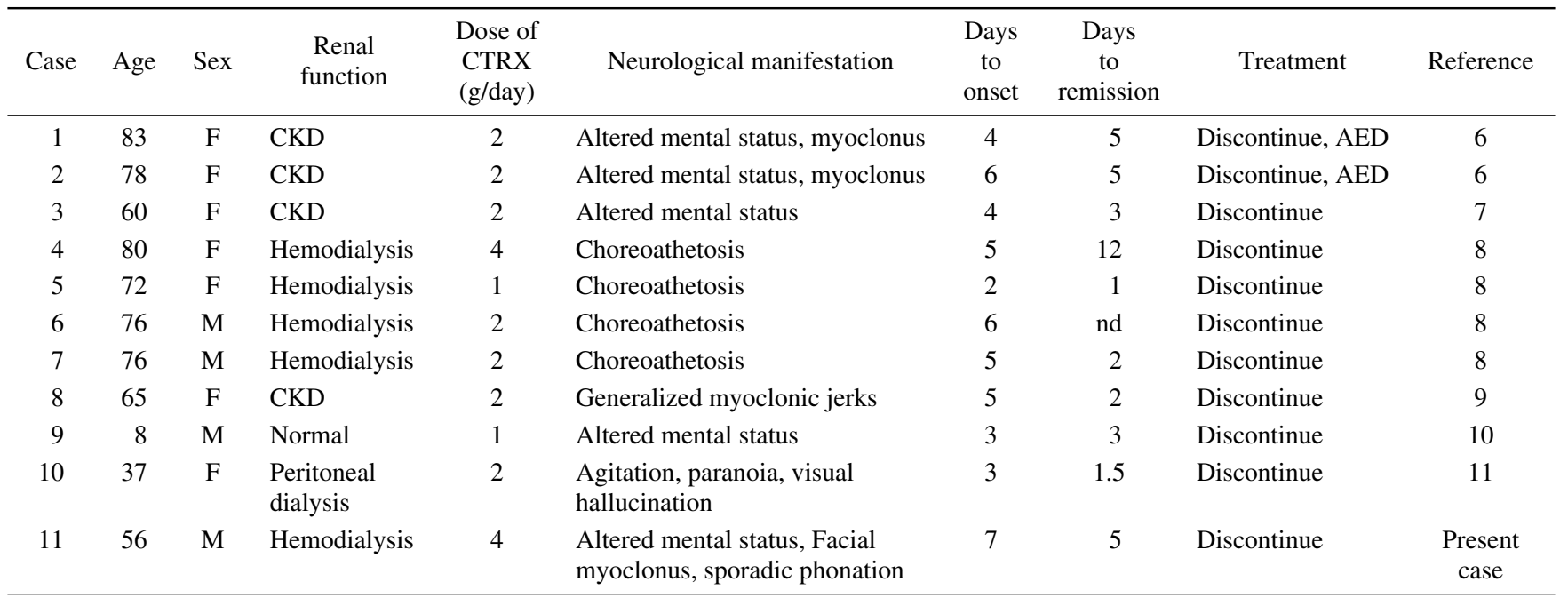

Neurological prognosis of the patients were all favorable.

AED: anti-epileptic drug, CKD: chronic kidney disease, nd: not described

myoclonus along with sporadic phonation. Third, radiographic and cerebrospinal fluid examinations do not provide any useful information for the diagnosis. Findings based on EEGs, although not specific, may aid in the diagnosis.

Patients' clinical courses provide the strongest basis for the diagnosis of AAE. The time spans to both the onset and amelioration of AAE have been reported to be 5 days in the median for most offending antibiotics (14). In previous cases of CTRX-induced neurotoxicity, the administration periods before the onset of neurological symptoms were 2 to 6 days, and clinical improvement was seen 1 to 12 days after cessation of the drug (9). In our case, the patient developed neurologic symptoms 7 days after initiation and recovered 5 days after the discontinuation of the drug. Rigorous documentation and observation of the patient's course can therefore lead to a definitive diagnosis of CTRX-induced neurotoxicity.

Specific treatment for CTRX-induced neurotoxicity may not be available. Hemodialysis is ineffective, as CTRX binds well to serum albumin and therefore cannot be removed from the blood (1). As such, discontinuation of the offending drug following an early diagnosis is crucial (14).

In conclusion, this report illustrated a case of CTRXinduced transient encephalopathy that involved a patient on hemodialysis. Although CTRX is a widely available and potent antibiotic in various clinical settings, clinicians need to be aware that patients with an impaired renal function may develop neurotoxicity. The clinical manifestations may be subtle or nonspecific, and therefore, the diagnosis of the adverse event is challenging; however, the early recognition followed by the discontinuation of the causal drug can lead to a rapid recovery. Close observation of a patient's clinical course can provide essential cues regarding the diagnosis.

Informed consent was obtained from the patient for the publication of this case.
The authors state that they have no Conflict of Interest (COI).

\section{References}

1. Patel I, Sugihara J, Weinfeld R, Wong E, Siemsen A, Berman S. Ceftriaxone pharmacokinetics in patients with various degrees of renal impairment. Antimicrob Agents Chemother 25: 438-442, 1984.

2. Lam S, Gomolin IH. Cefepime neurotoxicity: case report, pharmacokinetic considerations, and literature review. Pharmacotherapy 26: 1169-1174, 2006.

3. Kim S-Y, Lee I-S, Park SL, Lee J. Cefepime neurotoxicity in patients with renal insufficiency. Ann Rehabil Med 36: 159-162, 2012.

4. Chow K, Hui A, Szeto C. Neurotoxicity induced by beta-lactam antibiotics: from bench to bedside. Eur J Clin Microbiol Infect Dis 24: 649-653, 2005.

5. Grill MF, Maganti R. Cephalosporin-induced neurotoxicity: clinical manifestations, potential pathogenic mechanisms, and the role of electroencephalographic monitoring. Ann Pharmacother 42: 1843-1850, 2008.

6. Martínez-Rodríguez JE, Barriga FJ, Santamaria J, et al. Nonconvulsive status epilepticus associated with cephalosporins in patients with renal failure. Am J Med 111: 115-119, 2001.

7. Roncon-Albuquerque Jr R, Pires I, Martins R, Real R, Sousa G, von Hafe P. Ceftriaxone-induced acute reversible encephalopathy in a patient treated for a urinary tract infection. Neth J Med 67: 72-75, 2009 .

8. Sato Y, Morita H, Wakasugi H, et al. Reversible chreoathetosis after the administration of ceftriaxone sodium in patients with endstage renal disease. Am J Med Sci 340: 382-384, 2010.

9. Kim KB, Kim SM, Park W, Kim JS, Kwon SK, Kim H-Y. Ceftiaxone-induced neurotoxicity: case report, pharmacokinetic considerations, and literature review. J Korean Med Sci 27: 11201123, 2012.

10. Sharma N, Batish S, Gupta A. Ceftriaxone-induced acute reversible encephalopathy in a patient with enteric fever. Indian J Pharmacol 44: 124, 2012.

11. Safadi S, Mao M, Dillon JJ. Ceftriaxone-induced acute encephalopathy in a peritoneal dialysis patient. Case Rep Nephrol 2014: 108185, 2014.

12. Marco S, Cecilia F, Patrizia B. Neurologic complications after 
solid organ transplantation. Transpl Int 22: 269-278, 2009.

13. Pruitt AA, Graus F, Rosenfeld MR. Neurological complications of solid organ transplantation. Neurohospitalist 3: 152-166, 2013.

14. Bhattacharyya $S$, Darby RR, Raibagkar P, Castro LNG, Berkowitz AL. Antibiotic-associated encephalopathy. Neurology 86: 963-971, 2016.

15. Wallace KL. Antibiotic-induced convulsions. Crit Care Clin 13: 741-762, 1997.

16. Schliamser SE, Cars O, Norrby SR. Neurotoxicity of B-lactam antibiotics: predisposing factors and pathogenesis. J Antimicrob Che- mother 27: 405-425, 1991.

17. Calandra G, Lydick E, Carrigan J, Weiss L, Guess H. Factors predisposing to seizures in seriously ill infected patients receiving antibiotics: experience with imipenem/cilastatin. Am J Med 84: 911918, 1988.

The Internal Medicine is an Open Access article distributed under the Creative Commons Attribution-NonCommercial-NoDerivatives 4.0 International License. To view the details of this license, please visit (https://creativecommons.org/licenses/ by-nc-nd/4.0/).

(C) 2017 The Japanese Society of Internal Medicine Intern Med 56: 3103-3107, 2017 